\title{
A Note on the Treatment of some of the Commoner Dermatological Conditions.
}

\author{
R. M. B. MacKenNa.
}

T $\mathrm{T}$ has been truly said, in regard to medicine, that " life is short, and the art long, experience fallacious, and judgment difficult," and many will readily agree that in no branch of the art is experience more fallacious or judgment more difficult than in the treatment of dermatological conditions. The ointment which will cure a given case of eczema will but serve to aggravate an apparently similar case of the same disease, and almost in self defence the general practitioner has fallen back on calamine lotion and Lassar's paste as the two standard remedies which are least likely to aggravate an inflamed skin, and which may be relied upon to cure the majority of the simpler skin diseases.

Calamine lotion and Lassar's paste are both excellent standard remedies, but, unfortunately, "stock recipes" (although one has to rely on them when handling large numbers of patients in hospital) are of little use in dermatology. There is no " Digitalis mixture " in a hospital pharmacopæia, for the dose of digitalis has to be determined for each individual patient; similarly each ingredient in a lotion or ointment must be chosen deliberately for its therapeutic action, and the amount of each ingredient should be a matter of deliberation, and must vary for each case. It follows, therefore, that a true knowledge of the therapeutics of dermatology can only be acquired with some difficulty and by long practice, but the acquisition may be facilitated by the memorisation of the four following principles which form the foundation of a rational method of treating skin diseases :

(1) Soothe and cool a hot inflamed skin by the application of lotions or powders. tion.

(2) Never apply ointment to an oozing skin condi-

(3) Stimulate chronic eruptions.

(4) If the skin is dry and harsh, lubricate it with oils or fats.

There are, of course, exceptions to these rules, but let us consider the treatment of a few diseases in the light of these generalisations :

Firstly, Impetigo, which, in its commonest form, is an acute infection of the skin with streptococci (streptococcus 
pyogenes being usually inculpated), and which is characterised by the effusion of a copious sero-purulent exudation from the affected area. If the usual mercurial ointment is applied on lint, it forms a waterproof covering under which the exudation spreads, thus infecting adjacent areas. Further, the lint and bandage prevent loss of heat from the covered area, the temperature of the skin rises, the openings of the sebaceous and sweat glands are dilated, and a very fertile soil is prepared in which the streptococci may increase and multiply in warmth and comfort. If the ointment is applied on gauze the local temperature is not increased to such an extent, and therefore a dressing of gauze is preferable to one on lint, but the best method of treating impetigo is to apply a lotion frequently, and to keep the discharge from spreading over the adjacent skin. Good results may be obtained by the following line of treatment :

(1) Remove the crusts either by softening them with olive oil, or by the application for four or six hours of a starch and boracic poultice.

(2) Apply the following lotion six or eight times daily :-

$\begin{array}{ccccc}\text { Recipe }: \text { Sulph. precip. } & \ldots & \ldots & \text { gr. ii } \\ \begin{array}{l}\text { Ichthyol } \\ \text { Calaminæ prep. }\end{array} & \ldots & \ldots & \ldots & \text { gr. iii } \\ \begin{array}{l}\text { Zinci oxidi } \\ \text { Glycerini }\end{array} & \ldots & \ldots & \ldots & \text { gr. xx } \\ \text { Glycer } & \ldots & \ldots & \text { m. xx } \\ \text { Aq. calcis } & \ldots & \ldots & \ldots & \text { dr. iv } \\ \text { Aq. ad. } & \ldots & \ldots & \ldots & \text { oz. i }\end{array}$
hours.

(3) Remove the crusts regularly once every thirty-six

(4) When the exudation ceases, and the only symptom is slight redness of the affected areas, apply the following ointment twice daily :

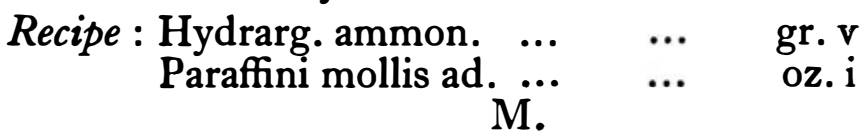

It will be seen that the rationale of this line of treatment is to soothe the inflamed areas with a modified calamine lotion, to increase the formation of crusts, so that the exudation is localised, and to apply the lotion so frequently that the ichthyol and the sulphur, and to a lesser extent, the other ingredients, can exert their antiseptic action on every drop of exudate.

The word " eczema" is usually employed to indicate an acute, subacute, or chronic inflammation of the skin, the 
etiology of which is unknown. The word is used to cover a multitude of inexactitudes in diagnosis, but the average case may be schematically represented thus :-

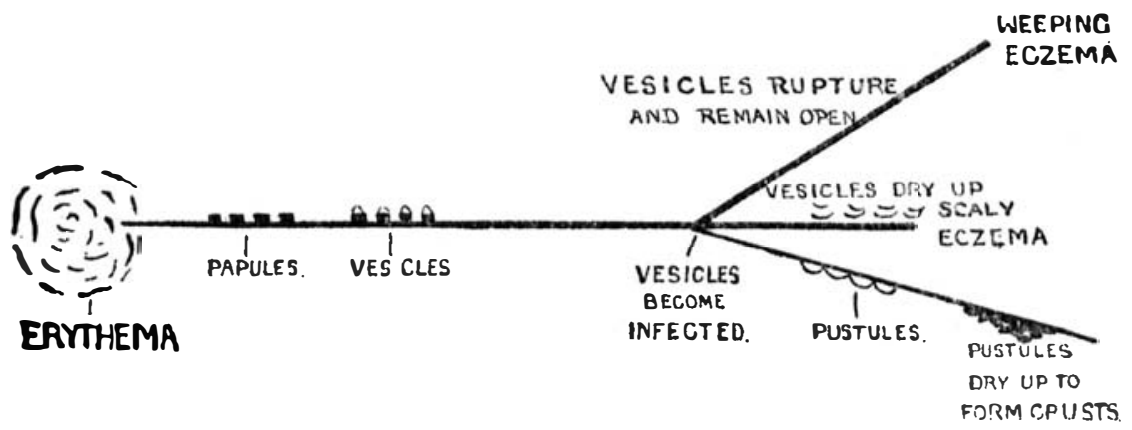

From R. W. McKenna "Diseases of the Skin" (Baillière, Tindall and Cox).

The condition begins with an erythema, and within a relatively short time vesicles form. The aftermath depends. on the progress of the vesicles.

Having regard to our principles, the treatment must vary in accordance with the stage of the disease. In the erythematous, papular and vesicular stages, the eruption must be cooled and soothed, and the following recipes will be of service :-

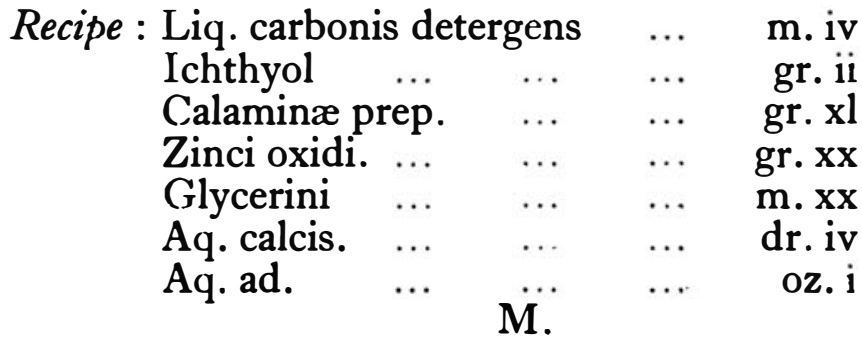

Recipe : Zinci oxidi ... $\quad \ldots \quad \ldots$ gr. $\mathrm{xxx}$

Liq. plumbi subacet. fort. ... m. iii

Glycerini $\quad \ldots \quad \ldots \quad \ldots \quad$ m. $x x$

$\begin{array}{llllr}\text { Ac. ad. } & \cdots & \cdots & \cdots & \text { oz. i }\end{array}$

Recipe : Pulv. acidi borici $\quad \ldots \quad \ldots \quad$ gr. $\mathrm{xv}$

Pulv. zinci oxidi $\quad \ldots \quad$... $\quad$ dr. iii

Pulv. talc. venet. ad. $\quad \ldots \quad$ oz. i

M.

It should be noted that the lotions are best applied on gauze, and that no oil silk, lint, wool, or gamgee tissue should be inserted between the gauze and the bandage. The lotions can then slowly evaporate, thus keeping the area under the: 
dressing cool, and helping to relieve the irritation which is the prominent symptom in these early stages.

The pustular and "weeping" stages are best treated with mildly astringent lotions, e.g., calamine lotion with 1 per cent. ichthyol, and as the acute condition subsides thin ointments or creams should be used, e.g. :-

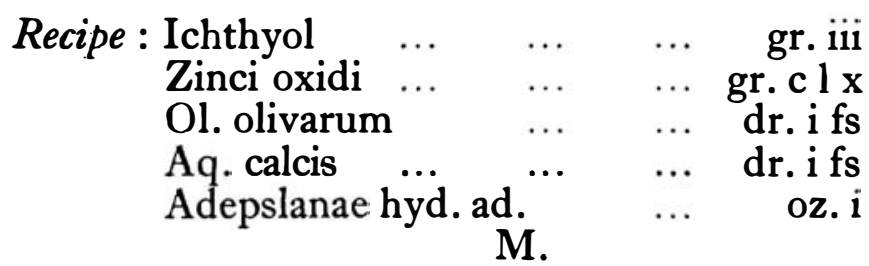

Chronic, dry, scaly eczema should be treated energetically, e.g.

Recipe : Liq. carbonis detergens _.. oz. iv.

Sig.: Add dr. i to half a pint of warm water and use to bathe the affected part for three minutes before applying the paste.

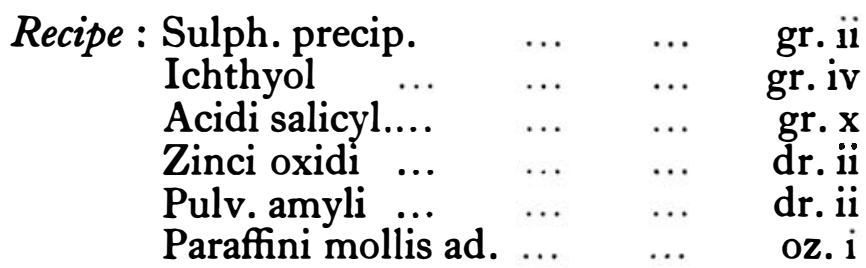

\section{M.}

Sig. : The paste. Apply twice daily.

It will be noted that many of the above prescriptions contain ichthyol. There are many varieties of this preparation on the market, and I have found the majority, which are synthetic preparations, to be unreliable and irritating. It is, therefore, wisest to specify a reliable brand. The ichthyol manufactured by Crookes, and the original German preparations are, in my opinion, the best.

Eczematous conditions of the external auditory meatus are often extremely resistant to treatment. The following well-known prescription has stood the test of time :-

$$
\begin{array}{cccc}
\text { Recipe : Acidi carbol liq. } & \ldots & \ldots & \text { m. iv. } \\
\text { Glycerini ad. } & \ldots & \ldots & \text { oz. fs. }
\end{array}
$$

Excellent results, however, may be obtained with a perchloride of mercury lotion applied on plugs for half an hour thrice daily, after which the meatus is dried and a little calamine lotion or zinc ointment applied. The recipe for the mercurial lotion is as follows :- 
Recipe : Hydrarg perchlor. Glycerini $\quad \ldots \quad \ldots$

gr. $1 / 6$

Aq.ad.

M.

m. $\mathbf{x x}$

dr. i

It is almost useless to generalise in regard to internal treatment, and dietetics, in the treatment of acute dermatoses. Arsenic, iron, quinine, intestinal antiseptics and polyglandular preparations must all be employed in accordance with general principles. Too frequently, in actual practice, the dietary prescribed varies more with the pre-conceived notions of the practitioner than with the actual requirements of the patients. In the majority of acute dermatoses and in cases of furunculosis it is advisable to instruct the patient to avoid taking sweet, sour, spicy and salty foods ; the four "s's" form a convenient mnemonic easily remembered both by the practitioner and the patient, and restrict the latter to a simple, but adequate diet, which fulfils most of the required desiderata.

With regard to alcohol, most patients suffering from acute or chronic skin diseases are much better without it, but if they insist on being allowed to take it, let them keep to the lightest French white wines. It is interesting to note that many psoriasis patients can bring out a florid eruption by a temporary and not immoderate indulgence in alcohol.

Furunculosis may, perhaps, be mentioned here in order to present a slightly different line of treatment than that usually employed by the surgeons. Boils may frequently be made to abort by painting them with a varnish made of equal parts of ichthyol and water, or by applying pure ichthyol on a whisp of cotton wool to the lesion. If abortion does not occur, the surrounding skin should be shaved and covered with ung. hyd. nit. dil., and hot fomentations may then be applied without the risk of adjacent pilo-sebaceous follicles being infected under the dressings. The boil should be incised as soon as central necrosis occurs, and the contents expressed by gentle pressure, or removed by means of a Bier's suction glass. Hot fomentations may be discontinued thereafter, and the following preparation (which, incidentally, is also an excellent remedy for bed sores and varicose ulcers) should be applied on lint thrice daily :

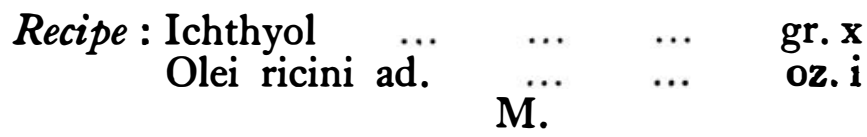


Personally, I have found the local application of " antivirus "solutions to be of but little value in the treatment of urunculosis.

One to three tablets of Stannoxyl (a proprietary preparation containing oxide of tin) may with advantage be taken thrice daily by the patient, as well as an iron and quinine tonic. Intramuscular injections of butyrate of manganese ( 1 to $1 \frac{1}{2}$ c.c.), or collosol manganese ( $\frac{1}{2}$ to 2 c.c.) given once every five days, are of the greatest value. They markedly increase the resistance of the patient to staphyloccocal infections ; they may be administered during the acute stages whilst he is in a " negative phase," and they have a stimulating effect on the organism. Unlike vaccines, they may, therefore, be given in the most acute stages; they do not produce the depression and lethargy which so frequently follows a course of vaccine.

In cases of furunculosis, complicating diabetes, or albuminuria, small injections of manganese preparations may be given without risk, and it may perhaps be noted here that these preparations are excellent remedies in the treatment of erysipelas, and in many instances are preferable to polyvalent antistreptococcal serum.

The fourth principle, given on an earlier page, viz.: that dry and harsh skins should be lubricated with oils and fats need scarcely be enlarged upon. It should be remembered when treating the later phases of an acute seborrhœic dermatitis, when an ointment containing ung. aqua rosæ and ung. zinci oxidi. is frequently of use, viz. :-

$$
\begin{array}{rlccc}
\text { Recipe }: & \text { Sulph. precip. ... } & \ldots & \ldots & \text { gr. iii } \\
\text { Hydrarg. ammon. } & \ldots & \ldots & \text { gr. ii } \\
& \text { Acidi salicye. ... } & \ldots & \ldots & \text { gr. } x \\
& \text { Pulv. amyli. } \ldots & \ldots & \ldots & \mathrm{dr} . \mathrm{i} \\
& \text { Ung. zinci oxidi } & \ldots & \ldots & \mathrm{dr} . \mathrm{iv} \\
& \text { Ung. aq. rosæ ad. } & \ldots & \ldots & \text { oz. i }
\end{array}
$$

M.

And in the chronic, dry, scaly condition, known as Ichthyosis, considerable relief may be obtained by the application of the following ointment :-

Recipe : Glycerini amyli.

Ung. acidi salicyl. aa partes æq.

$\mathrm{M}$.

Finally, I should like to discuss a parasitic disease, as an example of a condition in which none of the four principles mentioned above can be made to apply, and in which, in 
order to obtain a cure, substances which are irritating to the skin have to be used.

One of the few certainties in the whole realm of therapeutics is that sulphur will cure scabies. Unfortunately, sulphur ointment frequently produces a severe dermatitis, and it is, therefore, of ten advisable to dilute the ung. sulphuris of the British Pharmacopœia with two or three drachms of ung. zinci oxidi, to each ounce.

The usual method of treating scabies is for the patient to take a sulphur bath containing one-eighth of an ounce of potassa sulphurata to each gallon of water (a normal bath contains 25 to 30 gallons) on three successive nights, and after the bath to rub sulphur ointment into the affected parts. On the third night the bedding and clothes should be removed and disinfected, and the patient should be cured.

The S.P. Charges Co., of St. Helens, have two excellent preparations known as sulphaqua bath charges and unguentum thiomel. The former is a sulphur preparation to put in the bath, and it does not have the same deleterious action on the bath itself as " liver of sulphur." Ung. thiomel consists of sulphur in a honey-like base.

The use of these two preparations in conjunction is most satisfactory, as they cure the disease with a minimal risk of dermatitis.

The German firm of Bayer and Co. have recently put on the market a preparation known as "Mitigal." This will kill the sarcoptes scabiei, and will destroy its ova, and whilst being at least as efficient as sulphur ointment, does not seem to have the same proclivity of producing dermatitis.

The parasite which causes scabies is said only to be active during the night, and Norman Walker's suggestion that precipitated sulphur should be sprinkled between the sheets and over the body at night is therefore of value, for copulation between the male and female acari occurs on the surface of the skin, and the males do not burrow into the epidermis.

If the patient is unduly sensitive to sulphur, a 3 per cent. $\beta$ naphthol paste is excellent, viz. :-

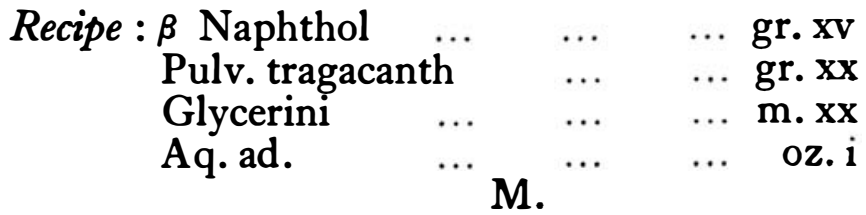

There remains one further line of treatment for scabies which should be mentioned here. It consists in the inunction 
of an ointment, the formula for which is extremely complicated. Marcussen discovered this cure in 1911, and Ehlers made it widely known in 1912. It was described in full in the British Medical Journal of January 25th, 1930, by A. Cannon, M.D., and is significant, because its protagonists claim that a single inunction of the ointment will cure the disease. The formula for the ointment is as follows :-

(a) $1 \mathrm{~kg}$. of sublimated sulphur is dissolved by heating it gently in $1 \mathrm{~kg}$. of a 50 per cent. solution of potassium hydroxide, a clear yellow solution resulting.

(b) Two hundred and twenty-five grammes of vaseline and 225 grammes of water-free lanolin are mixed with care, no heat being used.

(c) To this mixture 375 grammes of the solution of sulphur in potash lye [vide $(a)$ ] is added.

(d) Fresh zinc hydroxide is prepared by mixing 28 grammes zinc sulphate and 40 grammes of a 20 per cent. sodium hydroxide solution. Then this is added to the ointment.

(e) Liquid paraffin is added to make a total weight of 1,000 grammes.

( $f$ ) Five grammes of benzaldehyde is added to check the somewhat disagreeable smell of sulphuretted hydrogen.

The active principles in the ointment are the "high" sulphides of potassium, and such is their potency that certain dermatologists do not consider that it is necessary to disinfect the clothes and bedding after this treatment has been employed.

Cannon gives the following "procedure in treatment" :-

(1) The patient takes an ordinary cleansing bath.

(2) He then dries himself thoroughly.

(3) He applies the ointment to the whole of the body, except the head, by gentle rubbing only. Someone will assist him in anointing the back.

(4) He waits for twenty minutes, to allow the ointment to soak in.

(5) He then goes to bed, or does otherwise.

(6) The next lay, twenty-four hours after receiving the application of the ointment, the patient receives his second bath. cured.

(7) He puts on fresh underclothing, and walks away

I cannot claim to have any experience of this line of 
treatment, and can only say that a small quantity of the ointment made for me by a reliable firm of chemists deteriorated rapidly when kept in an ordinary gallipot.

The ointment, or a modification of it, is prepared by Zimmermann; it is named "Kathiolan" or Ung. hepatis sulfuris (Marcussen), and is packed in air-tight tins, but the method is worthy of considerable attention because it is known to have been of the utmost value in the treatment of the severe scabies common among the Norwegian lepers, which is believed to be due to infection with acari which are normally parasitic on wolves.

The whole of Dr. McKenna's useful article has been published, for apart from treating skin conditions in lepers, it will be of great value to nurses and others who have been perplexed regarding the treatment of skin conditions among European staffs and their families on their stations.

One or two points might be mentioned. Ordinarily in the native there is little fear of causing a sulphur dermatitis. The Danish treatment for itch mentioned by Dr. McKenna may not be practicable in certain institutions and camps, and the following treatment, which I have used, is very efficient :

$\begin{array}{llllllr}\text { Sulphur } & \ldots & \ldots & \ldots & \ldots & \ldots & 1 \text { part. } \\ \text { Lime } & \ldots & \ldots & \ldots & \ldots & \ldots & 1 \\ \text { Water } & \cdots & \ldots & \ldots & \ldots & \ldots & 10 \text { part. }\end{array}$

Boil down to five parts and cork well.

S. Apply with brush all over body. Make patient wash twenty-four hours later, and repeat this twice.

This mixture gives off sulphuretted hydrogen, and is a quick and efficient method of clearing up scabies among natives.

For the ordinary tinea (ringworm) crude coal tar is effective, or the following :
Crude coal tar
1 part.
Kerosine
2 parts.

Thia application is more irritating, and should not be used if septic conditions complicate the ringworm.

When an oily application is required, there is nothing better than crude hydnocarpus oil. When a boggy condition of the skin is found as a result of ringworm, complicated by scabies and eczema, then rubbing the patient with the following is very useful :

$$
\begin{array}{lllllll}
\text { Sulphur } \ldots & \ldots & \ldots & \ldots & \ldots & \ldots & \text { oz. iv } \\
\text { Hydnocarpus oil } & \ldots & \ldots & \ldots & \ldots & \ldots & \text { oz. } x
\end{array}
$$

EDITOR. 